not prejudicial to complete and early convalescence but rather the reverse. The patient is removed sooner from the infected zone and the risks of the many complications are minimised. Another advantage derived from this system is the increase of our hospital accommodation. This is, of course, a minor consideration, but from the point of view of a public official administering a large and expensive department it is a feature not to be disregarded. Can I not therefore claim that for the interests of public health, for the welfare of the patients, and for the advantage of the ratepayers, the system adopted has proved successful? It was not, I can assure you, without a feeling of considerable anxiety that I commenced to carry out a method so much at variance with the accepted traditions of the profession, but as case after case left the hospital without producing any of the dreaded results I felt to be working on safe grounds. Now, at the end of a year, I invite your opinion of the method employed.

Southampton.

\section{ABSTRACT OF A CONTRIBUTION TO THE STUDY OF THE ACTION OF INDIAN} COBRA POISON.' BY R. H. ELLIOT, M.B., B.S. Lond., F.R.C.S. ENG.,
D.P.H. CANTAB., MAJOR, I.M.S.

THIS research ${ }^{2}$ was undertaken by Major Elliot, who is on special duty making investigations into snake venom under the orders of the Secretary of State for India, with a view of accurately ascertaining the precise part played by the various important centres, nerves, and organs in the production of death from cobraism. After detailing the work of previous investigators and the methods employed in his researches Major Elliot came to the following summary of conclusions :-

1. Cobra venom acts directly on the muscular tissue of the blood-vessels or through their vasc-motor nerve endings, constricting the arterioles and thus raising the arterial blood pressure. It probably affects all organs alike. In the frog vessels the action can be traced down to dilutions of 1 in $10,000,000$. In a cobra bitten man the concentration of venom in the blood is probably at least 30 times as great as this.

2. Cobra venom also acts directly on the isolated frog ventricle, killing it in a position of firm systole if the solution be concentrated and stimulating it if a weaker strength be employed. The limit of the speedy lethal action on the isolated heart is reached at a concentration of about 1 in 500000 . The stimulating action can be traced down to a dilution of 1 in 10,000000 . This action of cobra venom brings it into line with the glucosides of the strophanthin group. Its action is more rapid than that of strophanthin and is certainly not inferior to it in strength. Atropin sulphate and cobra venom, when acting in the same solution, intensify each other's action and produce more summation of effect than one would have anticipated. This detracts from the value of the atropin salt in the treatment of cobraism and makes it a dangerous remedy. The blood pressure work has confirmed this view of the case.

3. Cobra venom powerfully affects the isolated mammalian heart when solutions of it are perfused through the coronary circulation. The action appears to be a dual one-viz. : (1) a direct action on the muscular fibre, or on the nerve endings, closely resembling that which is produced on the isolated frog ventricle; and (2) an action on the intracardiac vagal mechanism, which makes for inhibition. The result is that in strong solutions we find an irregular and extreme excitation of the heart, followed by early death in a position of systolic tone. If the concentration be less the early stage of excitement yields to a prolonged phase in which the

A paper communicated by Professor Sir Thomas R. Fraser, F.R.S., to the Royal Society on Feb. 25th, 1904.

2 Owin Professor E. A. Schäfer the writer was enabled to carry out this research in the Pharmacological and Physiological taboratories of the University of Edinburgh. Towards the expense of this research of the University of Edinburgh. Towards the expense of this research grants Moray Fund for the Endowment of Research (Edinburgh). tonic action of the poison on the heart is most pronounced; the beat is regular, steady, and strong. Cubra venom interferes with the circulation through the heart in a marked manner. This is probably due (1) to a constriction of the coronary vessels, brought about by the direct action of the venom on the ressel walls, and (2) to the condition of tonus into which the heart is tending to pass.

4. When given subcutaneously in low lethal doses cobra venom kills by paralysing the respiratory centre. Such a paralysis is in these circumstances gradually evolved and in the early stages of the process there is often evidence of a phase of stimulation preceding the paretic phase. There is a gradually increasing venosity of the blood and in consequence thereof all the harmtul resa'ts of slow asphyxiation are produced. If life is prolonged beyond the usual term by artificial respiration, and possibly also if the dose of venom is a very low lethal one which takes many hours to kill, the phrenic and other motor nerve ends may become paralysed, but this is certainly not an essential feature of death from lethal doses of cobra venom which kill within five hours. I hope to make a further communication on this subject later. The convulsions which precede death are purely asphyxial and can be at once stopped by artificial aeration of the blood. Each such convalsion is followed by a phase of exhaustion of the respiratory mechanism which is almost certainly central. If the dose of cotra venom administered be a large one, and especirlly if it be given intravenonsly, the respiratory centre is quickly and severely affected and respiration may cease almost at once. This cessation of breathing may be permanent if artificial re spiration be not quickly started, but if the dose be a smaller one the rhythmic activity of the centre reasserts itself. At first there may be a number of deep spasmodic gasps and then the movements of respiration re-begin, very gently at the commencement and gaining force as time goes on till a normal rhythm is re-established or even a stage of stimulation is manifested. Soon, however, the centre fails again and all the phenomena of asphyxiation appear. By applying cobra venom directly to the exposed medulla oblongata of the rabbit I have shown that the respiratory centre can be paralysed without the phrenic nerve ends or the heart being apprecially affected. If very large doses of venom are injected death may take place by cardiac failure before the respiratory mechanism has given way. We have here to do with the direct action of the venom on the heart muscle ; the beats become rapid and shortened and the heart passes into a systolic phase in which it dies tightly contracted.

5. Cobra venom when given in low lethal doses sub. cutaneously raises the general blood pressure. There may be a slight preliminary fall before the rise but often this is wanting. In the absence of further interference the blood pressure remains high till very near the end of life. In the asphyxial convulsions which herald death a further steep rise of blood pressure takes place; this is soon followed by a sudden and very rapid fall to death. The bigh level of blood pressure is due to (1) the direct action of the circulating venom on the muscular tissue of the arterioles, causing a constriction of these vessels and thus opposing a barrier to the onward flow of the blood; (2) the increased force of the heart beat as the outcome of the direct stimulating action of the venom on its muscular tissue ; and (3) the stimulation of the vaso-motor centre, as a result of the steadily increasing venosity of the blood. The slight preliminary fall of blood pressure which is sometimes seen is due to cardiac inhibition, bat this subject will be reserved for discussion when dealing in the next section with the action of large doses of the poison. The late fall in the rate of the heart beat is due to cardiac inhibition; the latter is due to several factors. 1. A gradually progressive asphyxiation is taking place throughout such an experiment; this affects the vagal centre in common with the rest of the nervous system; the result is a stimulation of the inhibitory mechanism and a consfquent slowing and weakening of the heart. 2. The direct stimulating action of the venom on the vagal inhibitory centre acts in the same direction as the asphyziation of the centre. 3 . There is distinct evidence that even when the irfluence of the vagal centres is removed inhibition of the heart continues to progress, though in a lessened degree. The obvious inference is that the vagal nerve ends are stimulated by the circulating venom and probably also as a result of deficient aeration of the blood. 4. It is not improbable that a stage of exhaustion of the heart muscle follows the early stimulative action of 
the venom. 5. Exhaustion of the heart is probably predisposed to by the strain put upon the organ in having to work for a long period against an abnormally high blood pressure.

We are now in a position to explain the sudden rapid fall of the curves of heart-beat rate and of blood pressure which ushers in death at the close of one of these long experiments. An over-strained and weakened heart is suddenly and violently called upon to bear a further burden, for respiration has ceased and the medullary centres are acutely asphyziated. As a consequence there is a violent excitation of the cardio-inhibitory and vaso-motor mechanisms. The heart is slowed and at the same time has to work against a suddenly increased pressure and it gives way. In fact, we have the phenomena of asphyziation in their entirety. The vessels of the splanchnic area are affected pari passu with those of the body generally and they in no wise act independently. The vaso-motor mechanism remains active throughout and is, as we have seen, profoundly affected by changes in the venosity of the blood.

6. Cobra venom, when injected in large doses and especially when given intravenously, causes: (1) a sudden fall of blood pressure; (2) a subsequent rise, provided that the dose has not been too large; and (3) a final fall to zero. The early fall is undoubtedly due to inhibition of the heart. It has been clearly shown that this is mainly brought about by the direct action of the poison on the vagal centres in the medulla oblongata, as it occurs before the accompanying failure of respiration has had time to act. Moreover, it is seen whilst artificial respiration is being actively carried on and can be checked in these circumstances by division of the vagi. On the other hand, there can be no doubt that asphyxiation of the vago-inhibitory centre intensifies and maintains the inhibition which direct influence of the venom on the vagal centre produces. The spontaneous recovery of respiration or the application of artificial respiration has a powerful influence in mitigating the action of the venom on the vagal centre. In the same way artificial respiration, and to a less extent the spontaneous recovery of respiration, appear to act beneficially on the poisoned respiratory centre. Even if the heart is cut adrift from all central vagal impulses, whether direct or indirect, by the division of the vagi there yet remains evidence of a continued inhibition which must be attributed to the direct action of cobra venom on the terminals of the vago-inhibitory mechanism. This action would appear to be a direct one but there is every probability that it is indirect as well, in other words, that it acts through asphyxiation of the vagal terminals as well as by the poisoning of these parts by the circulating poison. There is, however, another factor which must not be lost sight of-viz., a direct exhaustion of the heart muscle as the result of irregular over-stimulation.

7. When the secondary rise of blood pressure which follows the primary fall takes place it is due to the same factors which determine its occurrence when small doses have been injected. It remains to explain why it is sometimes absent brief, or ill marked. The explanation is simple : it is merely a question of cardiac failure. We have seen that the direct inhibitory action of the venom through the vagal centre is capable of overcoming the tendency which the blood circnlating through the heart muscle has to throw that muscle into death in systolic tone. Were it not for these two rival forces to some extent equilibrating each other cobra poison would kill by its direct action on the hear muscle. When the doses are comparatively small, or when the vagi are cut or thrown out of gear by atropin, we find the tonic cardio-muscular influence of the venom in evidence, but when the dose of venom is large, and especially when it is given intravenously (the vagi remaining intact), the inhibitory action overpowers the muscular excitation and failure of the heart occurs. If the inhibition is sufficiently well marked no amount of arteriolar spasm that occurs will compen ate it, consequently the blood pressure falls. When the dose of venom is a very large one the direct muscular stimulation may be so intense as to overcome the maximum inhibitory impuise and then the heart dies in systole with a quickened beat and is found after death as hard as a contracted post-partum uterus. In such circumstances any increase in the force of the heart is temporary, for the beat is probably a very partial one, the heart passes through a stage of excitement into one of increasing systolic tonus in which the contractions are very limited in extent.

\section{THE TREATMENT OF INCONTINENCE OF FACES BY THE SUBMUCOUS INJEC- TION OF PARAFFIN.'}

\section{By ARTHUR H. BURGESS, F.R.C.S. ENG., M.B.,} M.Sc. VICT.,

VISITING SURGEON TO THE MANCHESTER UNION HOSPITAL; SURGICAI OFFICER TO THE MANCHESTER CANCER PAVIIION.

INCONTINENCE of fæces may arise from many and very varied causes but from the point of view of surgical treatment the cases may be broadly classed into two groups. 1. Those cases in which the sphincter ani still retains some amount of power but not sufficient to close entirely the anal orifice. It is conceivable that if by introducing paraffin into the submucous tissue on the inner surface of the sphincter we narrow the lumen of the bowel at this situation and thereby diminish the work which the sphincter has to perform, even a weakened sphincter may be able firmly to occlude the remaining lumen and so to render the patient continent. 2. Those cases in which the sphincter is completely paralysed, as in many nervous diseases, or bas been entirely removed as after the operation of excision of the rectum. Various plastic procedures have from time to time been adrocated with the object of surrounding the anus in such cases with muscular fibres diverted from their natural function, the chief muscles concerned being the gluteus maximus and the levator ani. Attempts have also been made to produce a valvular action of the rectal wall, such as by dissecting out the rectum, twisting it on its long, axis, and refixing it, or by producing a sharp curvature or kink in its lower portion-attempts which are frequently unsuccessful. In two cases of incontinence of fæces belonging to this second group, one of which was associated with rectal prolapse, 1 have obtained great benefit from the submucous injection of paraffin into the lower portion of the rectum, as originally suggested by Gersuny of Vienna in 1899.

CASE 1.-The patient, a male, aged 58 years, was admitted to the Manchester Union Hospital with the history that three years previously he had undergone two operations elsewhere for fistula in ano, as the result of which the fistula was cured but he was completely incontinent of fæces. Two subsequent plastic operations were performed to relieve this condition, but were unsuccessful, and he had had absolutely no control over his stools for three years. On examination no trace of a sphincter could be discovered, the anus ex. tended in a posterior direction almost to the coccyx and was surrounded by several dense scars, the result of former ulceration. Under anæesthesia I injected paraffin in several places into the submucous tissue of the rectum, commencing as high up as I could reach and working towards the anus. In all, upon two occasions, the rather large amount of 52 cubic centimetres of paraffin was introduced, its melting point being $111^{\circ} \mathrm{F}$. This patient was shown at a clinical meeting of the Manchester Medical Society three weeks after the operation and I last heard of him nine weeks afterwards, during which interval he had had complete control over his motions and had never soiled his linen upon a single occasion.

CASE 2. - The patient, a boy, aged seven years, had been an inmate either of the Manchester Union Hospital or of the Swinton Schools attached thereto for the last five years, suffering from complete incontinence of fæces asso. ciated with a large rectal prolapse dating from infancy During the past four years he had undergone linear cauterisation on two or three occasions, excision of the ellipticai folds of the mucous membrane, and, finally, excision of th lower portion of the prolapsed rectum, all of which were unsuccessful in curing the prolapse, and had no effect upon the incontinence. The anus was abnormally large and lax and easily admitted four fingers, while there appeared to be an entire absence of sphincteric action. At first I injected only six cubic centimetres of paraffin submucously, but even this small amount certainly prevented the prolspse from descending with anything like its former frequency. On a subsequent occasion I introduced 27 cubic centimetres in three successive tiers, each tier consisting of three

1 A portion of a paper communicated to the Manchester Medical Society on Feb 3rd, 1904 . 